\title{
Carrier-carrier inelastic scattering events for spatially separated electrons: magnetic asymmetry and turnstile electron transfer
}

\author{
M.R. Poniedziałek and B. Szafran \\ AGH University of Science and Technology, \\ Faculty of Physics and Applied Computer Science, \\ al. Mickiewicza 30, 30-059 Kraków, Poland
}

(Dated: October 15, 2018)

\begin{abstract}
We consider a single electron traveling along a strictly one-dimensional quantum wire interacting with another electron in a quantum ring capacitively coupled to the wire. We develop an exact numerical method for treating the scattering problem within the stationary two-electron wave function picture. The considered process conserves the total energy but the electron within the wire passes a part of its energy to the ring. We demonstrate that the inelastic scattering results in both magnetic asymmetry of the transfer probability and a turnstile action of the ring on the electrons traveling separately along the ring. We demonstrate that the inelastic backscattering and / or inelastic electron transfer can be selectively eliminated from the process by inclusion of an energy filter into the wire in form of a double barrier system with the resonant energy level tuned to the energy of the incident electron. We demonstrate that the magnetic symmetry is restored when the inelastic backscattering is switched off, and the turnstile character of the ring is removed when the energy transfer to the ring is excluded for both transferred and backscattered electron waves. We discuss the relation of the present results to the conductance systems based on the electron gas.
\end{abstract}

PACS numbers: 73.63.-b, 73.63.Nm, 73.63.Kv

\section{INTRODUCTION}

During two past decades a significant progress in control and manipulation of separate electrons within the solid state devices has been made. A single electron was trapped within a quantum $\operatorname{dot}^{\underline{1}}$ in a localized state. Monitoring the flow of current by resolving the passage of separate electrons has been achieved $\stackrel{2}{2}$ An ultrafast single-electron pumping in a system of quantum dots connected in series was realized $\stackrel{3}{=}$ Single-electron AharonovBohm interference was demonstrated $\underline{\underline{4}}$ using a Coulombblockaded quantum dot as a valve injecting separate carriers into the channel via cotunneling events. Recently, single-electron transfer in a channel placed above the Fermi energy of the reservoirs was reported ${ }^{5}$ with the surface acoustic waves used to trap the moving carrier.

A single electron moving within the channel can be scattered inelastically and pass its energy to the environment. On the other hand for the conventional experiments with the electron gas, inelastic scattering of the Fermi level electrons is forbidden by the Pauli exclusion principle. The electron transport is strictly a Fermi level property in the linear regime, where the current $I$ is necessarily an even function of the external magnetic field $B$, i.e. $I(B, V)=G(B) V$, where $G$ is the linear conductance and $V$ the applied bias. The Landauer-Büttiker approach derives the linear conductance $G(B)=\frac{e^{2}}{h} T(B)$ out of the electron transfer probability $T$, and the latter is an even function of the magnetic field $T(B)=T(-B)$. The Onsager-Casimir ${ }^{6}$ symmetry $G(B)=G(-B)^{\underline{\underline{7}}}$ does not hold for the non-linear transport, $\underline{\underline{8}}$ where a finite energy window participates in the current flow. Asymmetry of conductance by the non-linear currents carried by the electron gas was studied both experimentally ${ }^{\underline{9}}-15$ and theoretically $\underline{\underline{8}, \underline{16}} \underline{\underline{24}}$ in a number of papers.

Here we consider a single electron injected into a quantum wire and its probability to pass through an interaction range of another electron confined in a quantum ring placed in neighborhood, close enough to allow the capacitive coupling, 2.,5 between the carriers. We find that this probability is asymmetric in $B$. We investigate the relation of the magnetic asymmetry with the inelastic scattering effects. We indicate that the magnetic symmetry of the electron transfer is restored when the inelastic backscattering is excluded. The latter is achieved by inserting a narrow band-pass energy filter in form of a double barrier structure into the channel with the resonant energy fixed at the energy of the incoming electron. We show that the energy filter introduced into the channel restores the magnetic symmetry of the transfer probability only for the electrons traveling in one direction and not the other, hence the turnstile character of the system is observed with or without the energy filter.

An appearance of the magnetic asymmetry of the single electron transfer probability was previously discussed in an bent quantum wire $\underline{25}$ or a cavity $\underline{26}$ asymmetrically connected to terminals. Both papers ${ }^{25,26}$ used a time dependent wave-packet approaches and indicated that the asymmetry of the transfer probability arises when the channel electron interacts with the surrounding environment. The present study of the role of the inelastic scattering requires a discussion of the incoming electron of a definite energy rather than the wave packet dynamics. We develop such an approach below and explain its relation to wave packet scattering. The results of this paper are based on a solution of the two-electron Hamiltonian eigenequation with an exact account taken for the interaction and the electron-electron correlations. 
This paper is organized as follows. In the next section we first sketch the two-electron Hamiltonian used in this paper in strictly one dimensional models of both the wire and the ring. Next, we present a time-dependent approach to the scattering problem and then the timeindependent treatment. We demonstrate that the results of the latter can be understood as the limit of monoenergetic wave packet scattering. Section III contains the results and IV the discussion. Summary and conclusions are given in section $\mathrm{V}$.

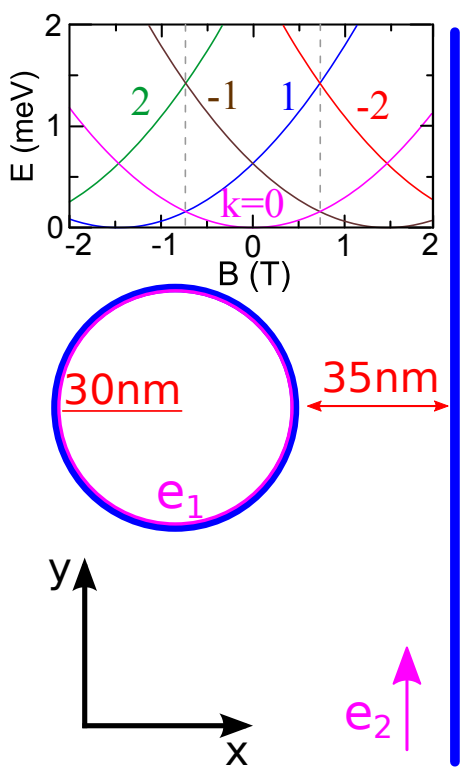

FIG. 1: Schematics of the considered system: the electron $e_{2}$ travels along a straight channel and is scattered on the potential of $e_{1}$ electron that is confined in a quantum ring of radius $30 \mathrm{~nm}$ placed at a distance of $35 \mathrm{~nm}$ from the channel. The top plot shows the energy spectrum of the electron in the ring.

\section{THEORY}

The system considered in this paper is schematically depicted in Fig. 1. An electron is confined in a circular quantum ring of radius $R=30 \mathrm{~nm}$. Initially, this electron is in its ground-state, with a definite angular momentum and circularly symmetric charge distribution. Another electron injected from outside goes along the straight channel, interacts with the ring-confined-electron and is partially backscattered. The total energy of the twoelectron system is a conserved quantity. The incoming electron is scattered inelastically when the ring absorbs a part of its energy.

The Hamiltonian of the electron in the circular ring with center in point $\left(x_{c}, y_{c}, 0\right)$ is given by $h_{r}=\frac{1}{2 m^{*}}(\mathbf{p}+$ $e \mathbf{A})^{2}+V\left(r_{c}\right)$ with $r_{c}^{2}=\left(x-x_{c}\right)^{2}+\left(y-y_{c}\right)^{2}$. The magnetic field $(0,0, B)$ is oriented perpendicular to the plane of electron confinement. For the symmetric gauge
$\mathbf{A}_{s}=\frac{B}{2}\left(-(y-y c), x-x_{c}, 0\right)$ the Hamiltonian of the ring electron takes the form $h_{r}=-\frac{\hbar^{2}}{2 m^{*}} \nabla^{2}+V\left(r_{c}\right)+\frac{e^{2} B^{2}}{8 m^{*}} r_{c}^{2}+$ $\frac{e B}{2 m^{*}} l_{c}$, where $l_{c}$ is the operator of the angular momentum $z$-component with respect to the ring center. Operators $h_{r}$ and $l_{c}$ have common eigenstates $\phi_{l}^{c}=f_{l}\left(r_{c}\right) \exp (i l \theta)$, with the angular momentum quantum number $l$. In the limit of a thin ring the radial wave function $f_{l}$ tends to the ground-state of a particle confined in an infinite quantum well and looses its dependence on $l$. The energy spectrum is then given by $\varepsilon_{l}=E_{r}+\frac{\hbar^{2}}{2 m^{*} R^{2}}\left(l+\frac{\Phi}{\Phi_{0}}\right)^{2}$ (see the inset to Fig. 1), where $\Phi_{0}=\frac{h}{e}$ is the flux quantum, $\Phi=B \pi R^{2}$ and $E_{r}$ is the ground-state energy of the radial confinement. The latter is independent of $l$ and as such is irrelevant for the scattering process. We skip $E_{r}$ in the following formulae.

For the scattering problem it is most convenient to use another gauge $\mathbf{A}=B(0, x, 0)$, since then the diamagnetic term produced by the kinetic energy operator $\left(\frac{e^{2} B^{2}}{8 m^{*}} x^{2}\right)$ vanishes at the axis of the channel $x=0$. In the following we assume that the channel is so thin that the electron in its motion along the channel is in its lowest state of lateral quantization. For the strictly 1D channel with $x=0$ axis the kinetic momentum $\pi_{y}=\mathbf{p}_{y}+e B x$ is independent of $B$, and thus the wave vector $q$ of the motion along the lead corresponds to the same energy and probability current flux for any $B$.

In order to replace $\mathbf{A}_{s}$ by $\mathbf{A}$ the gauge transformation $\mathbf{A}=\mathbf{A}_{s}+\nabla \chi(x, y)$ is performed with $\chi=\frac{B}{2}\left(x y+x_{c} y-\right.$ $\left.y_{c} x\right)$. Upon the transformation the ring wave functions change to

$$
\phi_{l}=\phi_{l}^{c}\left(r_{c}, \theta\right) \exp \left(-\frac{i e}{\hbar} \chi(x, y)\right)
$$

where the phase factor introduced by $\chi$ is independent of $l$. Although with $\mathbf{A}$ the angular momentum with respect to the ring center does not commute with the Hamiltonian, $l$ still remains a good quantum number for description of the ring eingestates.

With the assumptions explained above the twoelectron Hamiltonian used in this work reads

$$
H=h_{c}\left(\mathbf{r}_{1}\right)+h_{r}\left(\mathbf{r}_{2}\right)+W\left(\left|\mathbf{r}_{1}-\mathbf{r}_{2}\right|\right),
$$

where $h_{c}=-\frac{\hbar^{2}}{2 m^{*}} \frac{\partial^{2}}{\partial y^{2}}$ is the channel electron Hamiltonian and $W$ is the interaction potential. The latter is taken in the screened Coulomb form

$$
W(r)=\frac{e^{2}}{4 \pi \epsilon \epsilon_{0} r} \exp (-r / \lambda),
$$

with dielectric constant $\epsilon=12.9$ and the screening length $\lambda=500 \mathrm{~nm}$

\section{A. Time-dependent scattering picture}

The general form of the two-electron wave function can without a loss of generality be developed in the basis of 
product of single-particle eigenstates with definite angular momentum for the ring and the wave vector within the channel $q$

$$
\begin{aligned}
\Psi\left(\mathbf{r}_{1}, \mathbf{r}_{2}, t\right) & =\sum_{q l} c_{q l}(t) \Phi_{q}\left(\mathbf{r}_{1}\right) \phi_{l}\left(\mathbf{r}_{2}\right) \\
& =\sum_{l} \psi_{l}\left(\mathbf{r}_{1}, t\right) \phi_{l}\left(\mathbf{r}_{2}\right),
\end{aligned}
$$

where the partial wave packets are defined as

$$
\psi_{l}\left(\mathbf{r}_{1}, t\right) \equiv \sum_{q} c_{q l}(t) \Phi_{q}\left(\mathbf{r}_{1}\right) .
$$

The electrons occupying separate regions in space (the wire and the ring) are essentially distinguishable. Antisymmetrization of Eq. (6) does not affect any of the results presented below due to the complete separability of the electron wave functions $\frac{27}{2}$ For that reason we skipped the anti-symmetrization in the following.

One puts the wave function (5) into the Schrödinger equation $i \hbar \frac{\partial \Psi}{\partial t}=H \Psi$ and projects the result on the ring eigenstates, which leads to a set of equations for the partial wave packets

$$
i \hbar \frac{\partial \psi_{k}\left(y_{1}, t\right)}{\partial t}=\sum_{l}\left(\left[\varepsilon_{l}+h_{c}\right] \delta(k, l)+W_{k l}\left(y_{1}\right)\right) \psi_{l}\left(y_{1}, t\right),
$$

where $W_{k l}\left(\mathbf{r}_{1}\right)=\left\langle\phi_{k}\left(\mathbf{r}_{2}\right)\left|W\left(\left|\mathbf{r}_{1}-\mathbf{r}_{2}\right|\right)\right| \phi_{l}\left(\mathbf{r}_{2}\right)\right\rangle$. Note, that the phase factor due to the gauge transformation (11) is canceled in the evaluation of the interaction matrix $W_{k l}$.

In the time dependent calculation we take for the initial condition a Gaussian wave packet $\Psi_{l}(y, t)=$ $\sqrt{\frac{\Delta k}{2 \pi^{1 / 4}}} \exp \left(-\frac{\Delta k^{2}}{4}\left(y-y_{0}\right)^{2}+i q y\right)$, where $l$ corresponds to the ground-state angular quantum number, the average momentum $q>0$ and $y_{0}$ is far below the ring. For $k \neq l$, in the initial condition $\Psi_{k}=0$ is applied. Calculations are performed with a finite difference scheme for the channel of length $16 \mu \mathrm{m}$ with $\Delta y=2 \mathrm{~nm}$. The results converge when $|l| \leq 3$ ring eigenstates are included into the basis.

\section{B. Stationary description of the scattering}

The time-independent approach described in this section is suitable for treating the scattering for the incident electron of a definite energy. The stationary approach is also more computationally effective and does not require very large computational box since transparent boundary conditions can readily be applied. For $\Delta k=0$ the incoming electron has a definite momentum $\hbar q$, and a definite energy $E_{i}=\frac{\hbar^{2} q^{2}}{2 m^{*}}$, hence the total energy $E_{t o t}$ of the system is also a well-definite quantity $E_{t o t}=E_{i}+\varepsilon_{l}$, where $\varepsilon_{l}$ is the ring ground-state energy. Therefore, the two-electron wave function for the scattering satisfies the time-independent Schrödinger equation

$$
H \Psi\left(\mathbf{r}_{1}, \mathbf{r}_{2}\right)=E_{t o t} \Psi\left(\mathbf{r}_{1}, \mathbf{r}_{2}\right) .
$$

We use the form of the function

$$
\Psi\left(\mathbf{r}_{1}, \mathbf{r}_{2}\right)=\sum_{l} \psi_{l}\left(\mathbf{r}_{1}\right) \phi_{l}\left(\mathbf{r}_{2}\right),
$$

which is a time-independent counterpart of Eq. (5). Insertion of Eq. (9) into Eq. (8) followed by projection on a ring eigenstate gives a system of eigenequations for $\psi_{l}$,

$$
\sum_{l}\left(\left[\varepsilon_{l}+h_{c}\right] \delta(k, l)+W_{k l}(y)\right) \psi_{l}(y)=E_{t o t} \psi_{k}(y) .
$$

The electron in the ring is initially in its ground-state with angular momentum $l$ - as in the time independent picture. Therefore, the partial wave $\psi_{l}$ at the input side is a superposition of the incoming and backscattered waves $a \exp \left(i q_{l} y\right)+b \exp \left(-i q_{l} y\right)$. Since $\Psi$ is defined up to a normalization constant, at the bottom of the computational box $\left(3 \mu m\right.$ long) we simply set $\psi_{l}(0, y=0)=a+b=1$ as the boundary condition. After the solution of Eqs. (10) the values of the incoming $a$ and the backscattered $b$ amplitudes are extracted from the form $\psi_{l}$ along the lead.

The partial waves for $k \neq l$ appear only due to the interaction of the incoming electron with the ring, and they all correspond to the electron flow from the ring to the ends of the channels. Thus, far away above [below] the ring the partial wave functions corresponding to $k$-th angular momentum quantum number correspond to transferred [backscattered] electron and have the form of $c_{k} \exp \left(i q_{k} y\right)\left[d_{k} \exp \left(-i q_{k} y\right)\right]$, with $q_{k}=\sqrt{\frac{2 m^{*}}{\hbar^{2}}\left(E_{t o t}-\varepsilon_{k}\right)}$. For $E_{t o t}>\varepsilon$ the wave vector $q_{k}$ is real and the boundary condition $\psi_{k}(y+\Delta y)=$ $\psi_{k}(y) \exp \left(i q_{k} \Delta y\right)\left[\psi_{k}(y+\Delta y)=\psi_{k}(y) \exp \left(-i q_{k} \Delta y\right)\right]$ is applied at the top [bottom] end of the computational channel. For $E_{t o t}<\varepsilon$ the wave vector $q_{k}$ is imaginary and the wave function vanishes exponentially along the lead. The partial waves with imaginary $q_{k}$ are counterparts of the evanescent modes 28 for scattering in two-dimensional channels. For imaginary wave vectors we put zero for $\psi_{k}$ at the ends of the computational box. Upon solution of Eq. (10), the amplitudes $a, b, c_{l}, d_{l}$ are calculated. The total transfer probability is given by $T=\sum_{k} T_{k}$ with $T_{k}=\frac{\left|c_{k}\right|^{2}}{|a|^{2}} \frac{q_{k}}{q_{l}}$, similarly the backscattering probability is $R=\sum_{k} R_{k}$ with $R_{k}=\frac{\left|d_{k}\right|^{2}}{|a|^{2}} \frac{q_{k}}{q_{l}}$.

\section{RESULTS}

In Fig. 2 we plotted the electron transfer probability obtained by the time-independent method in function of the incident electron energy, for three values of the magnetic field. For $E_{i}<1 \mathrm{meV}$ the transfer probability vanishes and for $E_{i}>3 \mathrm{meV}$ the value of $T$ becomes close to 1 independent of $B$. Around $E_{i}=1.6 \mathrm{meV}$ a distinct asymmetry of $T$ as a function of $B$ is found. The insets displays the charge density within the ring calculated as $\rho\left(\mathbf{r}_{2}\right)=\int d \mathbf{r}_{1}\left|\Psi\left(\mathbf{r}_{1}, \mathbf{r}_{2}\right)\right|^{2}$. For $B=0.4 \mathrm{~T}$ the 


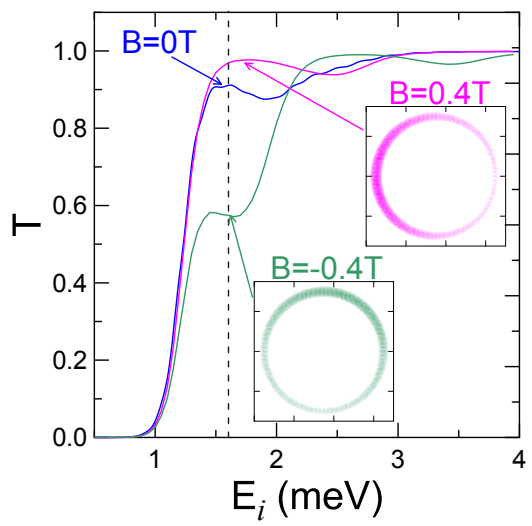

FIG. 2: Transfer probability of electron transport through channel in function of the incoming electron energy for three values of the magnetic field. The inset shows the charge density as obtained by the stationary transport description for three values of the magnetic field.

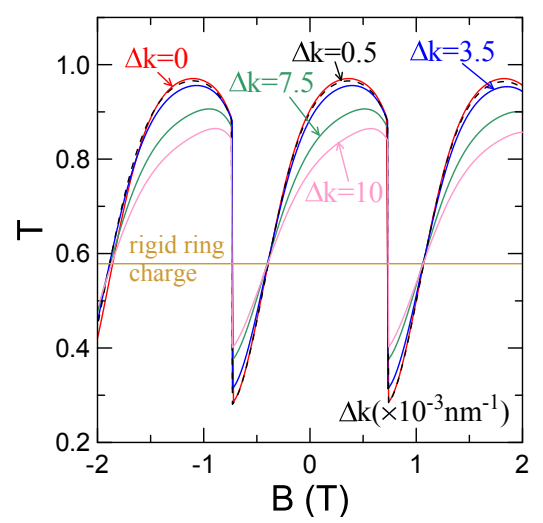

FIG. 3: The green, blue and black lines show the packet transfer probability through the system of Fig. 1 as calculated by the wave packet simulation for a number of wave vector dispersions for $\Delta k \leq 10^{-3} / \mathrm{nm}$ as functions of the external magnetic field. The red line shows the result of the time-independent scattering problem $(\Delta k=0)$. The horizontal line shows the transfer probability for fixed charge of the ring.

density is shifted off the channel (at right to the ring), and consistently $T$ is larger.

The results of the time-dependent simulation for the packet average energy of $\left\langle E_{i}\right\rangle=\frac{\hbar^{2} q^{2}}{2 m^{*}}=1.6 \mathrm{meV}$ are plotted in Fig. 3 in function of $B$ for a number of initial dispersions of the wave vector $\Delta k$. The horizontal line shows the result obtained for a rigid charge of the ring which is independent of $B$. All the $B$ dependence of the transfer probabilities given in Fig. 3 is due to the properties of the ring as an inelastic scatterer which change with the magnetic field. The discontinuities present in the transfer probabilities at $B= \pm B_{0}= \pm 0.73 \mathrm{~T}$ result from ground-state angular momentum transitions within the ring [see the top inset to Fig. 1]. With $\Delta k$ decreasing

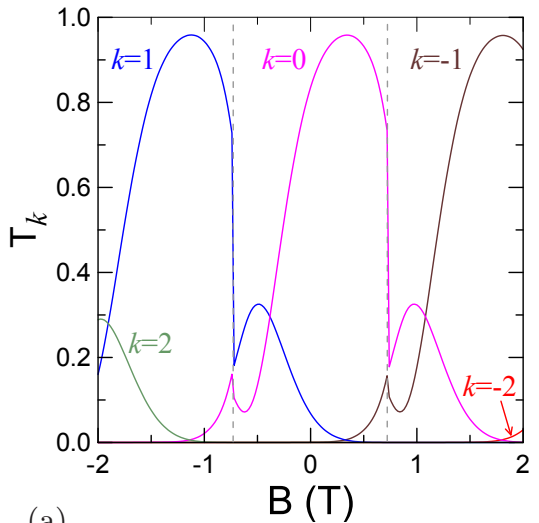

(a)

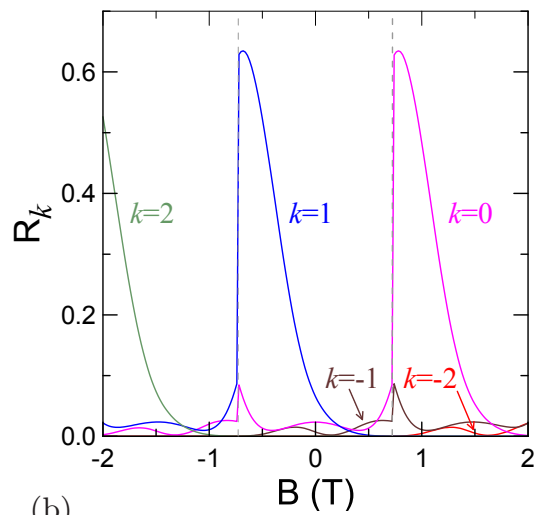

(b)

FIG. 4: Transfer (a) and backscattering (b) probability associated with angular momentum $k$ of the ring in the final scattering process (see text).

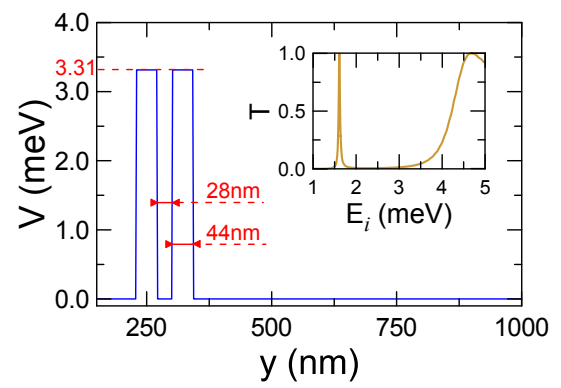

FIG. 5: Double barrier structure used a the energy filter. The inset shows transmission probability through the barrier in function energy with a peak at $1.6 \mathrm{meV}$ - the incident electron energy. The ring center is set at $y_{c}=1500 \mathrm{~nm}$.

to 0 the results converge to the result of the stationary description of the scattering for the incoming electron energy of definite energy $E_{i}=\frac{\hbar^{2} q^{2}}{2 m^{*}}=1.6 \mathrm{meV}$ which are plotted with the red line in Fig. 2. The rest of the results presented in this work was obtained with the stationary description of the transport.

The electron transfer probability as depicted in Fig. 3 is a distinctly asymmetric function of $B$. The asymmetry along with the character of the discontinuities at the ring ground-state transformations can be understood as due to the relation of the backscattering to the an- 


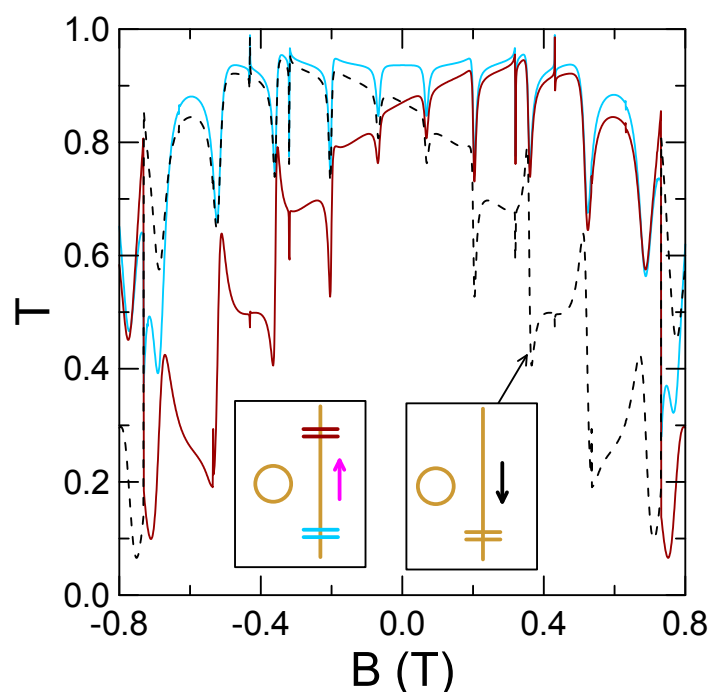

FIG. 6: Electron transfer probability for the DBS placed below (red curve) or above (blue curve) the ring for the electron incident from the lower end of the wire. The dashed curve shows the transfer probability for the electron going down with the DBS placed below the ring.

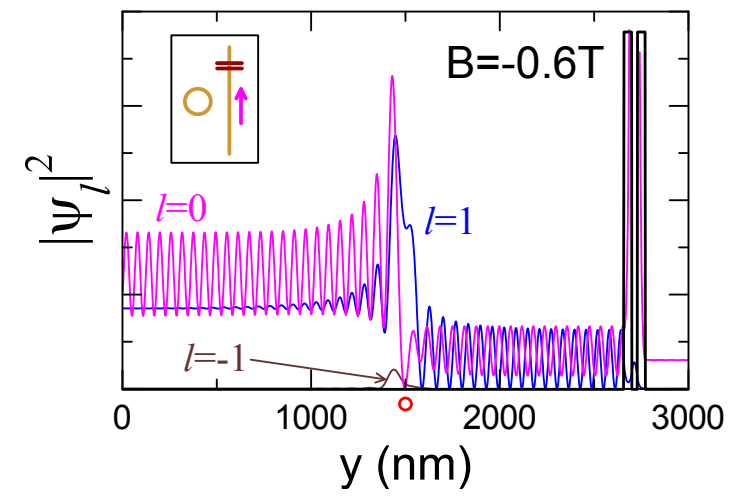

FIG. 7: Density of partial waves for the DBS placed above the ring. Location of the ring $(y=1500 \mathrm{~nm})$ is marked by a circle on the horizontal axis.

gular momentum absorption by the ring. The incoming (backscattered) electron has a positive (negative) angular momentum with respect to the center of the ring. When the ring electron compensates for the loss of angular momentum the backscattering is more probable. Let us concentrate on the magnetic field interval $\left[-B_{0}, B_{0}\right]$ in which the ring ground state corresponds to $l=0$. The absorption of the angular momentum by the ring is associated with transition from $l=0$ to $l=1$ energy level. This is less energetically expensive when $B$ becomes negative due to decreasing energy spacing between the ground state energy and the $l=1$ energy level (see the inset to Fig. 1). Consistently, the contribution of $l=1$ energy level to the total backscattering probability grows as $B$ decreases below 0 - see Fig. 4(b). Fig. 4(a) shows that for $B$ just above the ring-state transition $l=1$ ring state dominates also in the transfer probability.

Below the ground-state angular momentum transition which occurs at $B=-0.73 \mathrm{~T}$ the ring ground state $l$ is

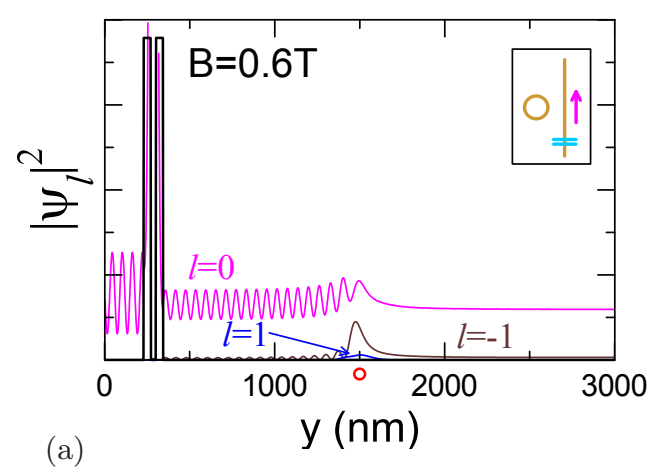

(a)

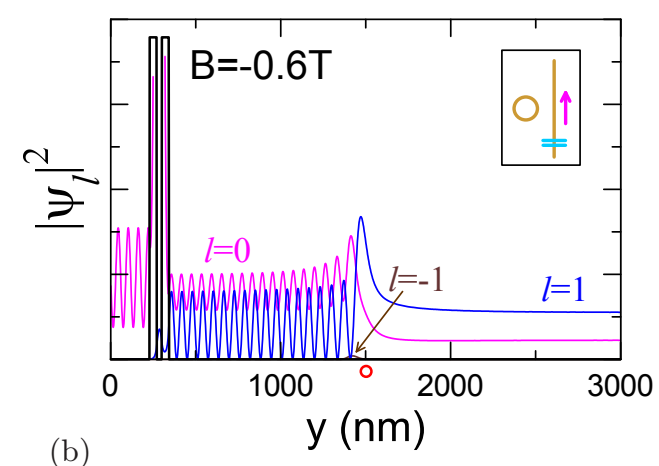

FIG. 8: Same as Fig. 7 only for the DBS placed below the ring.

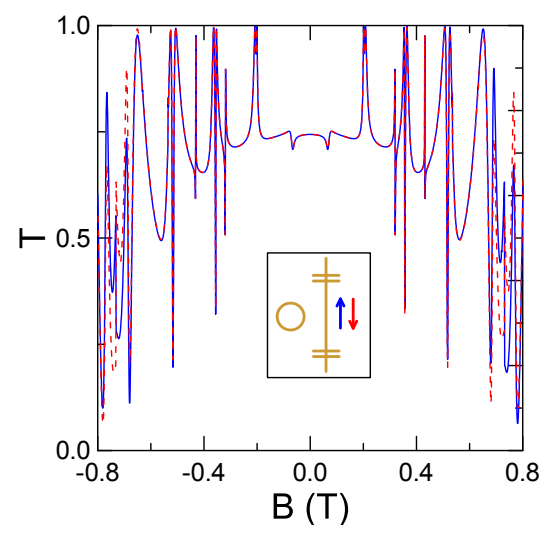

FIG. 9: Electron transfer probability for the DBS placed both below and above the ring for the electron going up (blue curve) or down (red dashed curve) the wire.

1 and the absorption of angular momentum by the ring requires an appearance of $l=2$ wave function to the final scattering process. This becomes energetically expensive below $B<-B_{0}$, hence the jump of $T$ that is observed in 


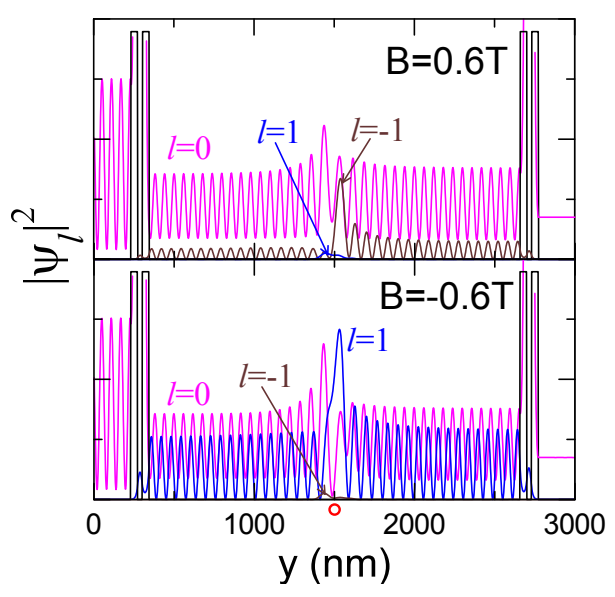

FIG. 10: Same as Fig. 7 only for two DBS: one below and second above the ring.

Fig. 3 at the ring ground-state transformation. As $B$ is decreased further $T$ drops and $l=2$ starts to dominate in the backscattering probability [see Fig. 4(b)].

Our results for the single-electron scattering indicate that the energy absorption is associated both with the electron transfer [Fig. 4(a)] and backscattering [Fig. $4(\mathrm{~b})$ ], which is accompanied by magnetic symmetry violation for the electron transfer probability. We found that one can eliminate selectively the effects of inelastic scattering in the transferred or backscattering waves by a proper tailoring of the potential profile along the channel. For that purpose we used a double barrier structure (DBS) with center placed on the channel far (1200 nm) below the ring. Figure 5 shows the applied potential profile and the inset to the figure the electron transfer probability through the DBS. We can see the resonant peak at the electron energy of $1.6 \mathrm{meV}$. The resonant energy was set equal to the energy of the incoming electron, so that the DBS acts like an energy filter - it is opaque for the electron that lost a part of its energy, i.e. to the partial waves with $k \neq l$.

In Fig. 6 we plotted with the red line the transfer probability for the DBS energy filter placed above the ring. Fig. 7 shows the plot of partial waves along the channel. Above the DBS one finds only the partial wave associated with $l=0$, i.e. with the ground-state of the ring. The electron can transfer across the structure only provided that the it preserves its initial energy. Therefore, no excitation of the ring electron is possible when the channel electron transfers across the structure. In Fig. 7 we can see that far below the ring we have an interference of $l=0$ incoming and backscattered waves. No interference is observed in the partial wave with $l=1$ near $x=0\left(\left|\psi_{1}\right|\right.$ is constant), since there is no incoming wave with $l=1$. Nevertheless an oscillation of $l=1$ wave is observed between the DBS and the ring. The potential of the ring and the DBS form a wide quantum well in which the partial waves [for instance $l=1$ in Fig. 7] oscillate back and forth. The presence of the wide well is also responsible for the resonances appearing at the $T(B)$ dependence in Fig. 6. $T(B)$ for the DBS placed above the ring remains an asymmetric function of $B$.

The transfer probability $T$ becomes an even function of $B$ (blue curve in Fig. 6) when the DBS energy filter is placed below the ring, which removes inelastically scattered partial waves of the total backscattered wave function. The partial wave function plots given in Fig. 8(a) and Fig. 8(b) show that below the DBS only the partial wave with $l=0$ is found, but above the structure we see an appearance of the partial waves for $l \neq 0$.

For $B>0$ just below $B_{0}$ we found that $T(B)$ is nearly the same for the double barrier structure placed both below and above the ring [see the blue and red curves which nearly coincide in Fig. 5 just below $B_{0}$ ]. Note, that for the DBS below the ring at $B=0.6 \mathrm{~T}$ we find that the contribution of $l \neq 0$ in the transferred wave function is negligible [Fig. 8(b)]. The absorption of the angular momentum by the ring is weak for $B \rightarrow B_{0}$ due to the large energy cost of this ring excitation [see the discussion of Fig. 2], hence the similar results found for both locations of DBS.

In Fig. 6 with the dashed curve we plotted the electron transfer probability for the DBS below the ring and the electron incident from the upper end of the wire. In this case the electron is first scattered by the ring and then by the DBS. We can see that for a single DBS present within the wire the transfer probability from one end of the wire to the other is different than in the opposite direction (the dashed curve in Fig. 6 can be obtained from the red one by inversion $B \rightarrow-B$ ), i.e. the system acts like a turnstile.

Figure 9 gives the electron transfer probability for two DBS placed both below and above the ring. The inelastic scattering is switched off for both the transferred and backscattered trajectories. The partial waves given in Fig. 10 show that the ring does get excited but only for the channel electron staying between the two DBS. We find that the transfer probability is symmetric with respect to both the magnetic field and the direction from which the electron comes to the ring. The small deviations off the symmetries visible at a closer inspection of Fig. 9 are due to small but finite width of the resonance peak (see the inset to Fig. 5). The inelastic scattering is allowed with the energy losses smaller than the width of the peak.

\section{DISCUSSION}

Results of Figs. 3 and 4 indicate that the asymmetry of the transfer probability as a function of the magnetic field is a result of 1) geometrical asymmetry of the system 2) inelastic electron scattering - the absorption of the angular momentum by the ring which is necessarily accompanied by the energy absorption 3) the energy transfer occurs through the electron-electron interaction.

For systems with the two-dimensional electron gas it was pointed out 8,17 that the magnetic asymmetry of conductance may result from the potential landscape within 
the device being not an even function of $B$ - the potential produced by charges at the edges of the channel in the Hall effect $\frac{8}{-}$ as the most basic example. In this case the asymmetry of the charge distribution is translated to the asymmetry of the transport by the electron-electron interaction. The role of the electron-electron interaction for the magnetic asymmetry of the transport in the electron gas was also indicated in Refs, $17,18,22$. In the present study of the single-electron transport the asymmetry is due to the properties of the ring - the enhancement of the backscattering accompanied by absorption of the angular momentum of the channel electron - which are not an even function of $B$ due to the form of the ring energy spectrum. Here, the backscattering is only due to the electron-electron interaction. Although in the linear transport regime the inelastic scattering of the electrons at the Fermi level is blocked by the fact that the states of lower energies are occupied, in the non-linear transport the inelastic scattering is not only allowed but necessary for thermalization of the carriers passing between electron reservoirs of unequal electrochemical potentials. The asymmetry that we find in this work results from the energy transferred by the channel electron to the ring, i.e. it occurs due to the inelastic scattering. The magnetic symmetry is restored when the inelastic backscattering is excluded. The invariance of the backscattering is invoked in explaining $T(B)=T(-B)$ symmetry when the transfer kinetics is very different for both magnetic field orientations - see the deflection of the electron trajectories by the Lorentz force in Ref. 25. In the present work the Lorentz force is excluded by the strict 1D approximation for the channels width. Nevertheless, the different kinetics resulting in the same transfer probability was also found in Figs. 8(a) and 8(b).

A single DBS placed below the ring restores the magnetic symmetry of the transfer, still only for the electron injected from one side of the channel and not the other (the microreversibility is not restored - see Fig. 6). Thus, for a single DBS present within the wire the electron transfer probability from one end of the terminal to the other are unequal. The turnstile character of the system is also a result of the inelastic scattering. The conditions present in the linear transport regime - with the inelastic scattering excluded at both the transfer and the backscattering - were simulated with two DBS placed at both sides of the ring. This configuration of energy filters restores the microreversibility of the system. The transfer probability becomes an even function of $B$, although the kinetics of the electron transfer is not identical for $\pm B$ [Fig. 10]. Moreover, the microreversibility is also restored [Fig. 9], although the system with two DBS is still not spatially symmetric under a point inversion.

\section{SUMMARY AND CONCLUSIONS}

We have studied single-electron scattering process on an electron localized in a quantum ring off the electron transport channel. We developed for that purpose a time-independent approach based on an expansion of the two-electron function in a basis of ring eigenstates and explained its relation to the numerically exact timedependent scattering picture. We have found that the electron transfer probability is an asymmetric function of $B$ and that the asymmetry results from the energy cost of the angular momentum absorption by the ring which is not an even function of $B$. We have demonstrated that the symmetry is restored when the electron backscattering with the energy loss is excluded. The exclusion was performed by a double barrier structure with the resonant state set at the energy of the incoming electron. In order to remove the turnstile character of the ring as a scatterer one needs to employ a pair of double barrier structures at both the entrance and the exit to the ring interaction range.

Acknowledgements This work was performed supported by the Polish Ministry of Science an Higher Education (MNiSW) within a research project N N202 103938 for 2010-2013. Calculations were performed in ACK-CYFRONET-AGH on the RackServer Zeus.
1 S. Tarucha, D.G. Austing, T. Honda, R.G. van der Hage, and L.P. Kouvenhoven, Phys. Rev. Lett. 77, 3613 (1996).

2 S. Gustavsson, R. Leturcq, B. Simovic, R. Schleser, T. Ihn, P. Studerus, K. Ensslin, D.C. Driscoll, and A.C. Gossard, Phys. Rev. Lett. 96, 076605 (2006).

3 S.P. Giblin, S.J. Wright, J.D. Fletcher, M. Kataoka, M. Pepper, T.J.B.M. Janssen, D.A. Ritchie, C.A. Nicoll, D. Anderson, and G.A.C. Jones, New. J. Phys. 12, 073013 (2010).

${ }^{4}$ S. Gustavsson, R. Leturcq, M. Student, T. Ihn, K. Ensslin, D.C. Driscoll, and A.C. Gossard, Nano Lett. 8, 2547 (2008).

5 R.P.G. McNeil, M. Kataoka, C.J.B. Ford, C.H.W. Barnes, D. Anderson, G.A.C. Jones, I. Farrer, and D.A. Ritchie,
Nature 477, 439 (2011)

${ }^{6}$ L. Onsager, Phys. Rev. B 38, 2265 (1931); H.B.G. Casimir, Rev. Mod. Phys. 17, 343 (1945);

7 M. Büttiker, Phys. Rev. Lett. 57, 1761 (1986); C.W.J. Beenaker, Rev. Mod. Phys. 69, 731 (1997).

8 D. Sánchez and M. Büttiker, Phys. Rev. Lett. 93, 106802 (2004).

9 G.L.J.A. Rikken and P. Wyder, Phys. Rev. Lett. 94, 016601 (2005).

10 J. Wei, M. Shimogawa, Z. Wang, I. Radu, R. Dormaier, and D.H. Cobden, Phys. Rev. Lett. 96, 116801 (2006).

11 R. Leturcq, D. Sánchez, G. Götz, T. Ihn, K. Ensslin, D.C. Driscoll, and A.C. Gossard, Phys. Rev. Lett. 96, 126801 (2006). 
12 D.M. Zumbühl, C.M. Marcus, M.P. Hanson, and A.C. Gossard, Phys. Rev. Lett. 96, 206802 (2006).

13 A.D. Chepelianskii and H. Bouchiat, Phys. Rev. Lett. 102, 086810 (2009).

14 B. Brandenstein-Köth, L. Worschech, and A. Forchel, Appl. Phys. Lett. 95, 062106 (2009).

15 S. Nakamura, Y. Yamauchi, M. Hashisaka, K. Chida, K. Kobayashi, T. Ono, R. Leturcq, K. Ensslin, K. Saito, Y. Utsumi, and A.C. Gossard, Phys. Rev. B 83, 155431 (2011).

16 B. Spivak and A. Zyuin, Phys. Rev. Lett. 93, 226801 (2004).

17 M. Büttiker and D. Sánchez, Int. J. Quantum Chem. 109, 906 (2005).

18 E. Deyo, B. Spivak, A. Zyuzin, Phys. Rev. 74, 104205 (2006).

19 M.L. Polianski and M. Büttiker, Phys. Rev. Lett. 96, 156804 (2006); Phys. Rev. B 76, 205308 (2007).
20 A. De Martino, R. Egger, and A.M. Tsvelik, Phys. Rev. Lett. 97, 076402 (2006).

21 A.V. Andreev and L.I. Glazman, Phys. Rev. Lett. 97, 266806 (2006).

22 D. Sánchez and K. Kang, Phys. Rev. Lett. 100, 036806 (2008).

23 A.R. Hernández and C.H. Lewenkopf, Phys. Rev. Lett. 103, 166801 (2009).

24 J.S. Lim, D. Sánchez, and R. López, Phys. Rev. B 81, 155323 (2010)

25 R. Kalina, B. Szafran, S. Bednarek, and F.M. Peeters, Phys. Rev. Lett. 102, 066807 (2009).

26 B. Szafran, M.R. Poniedziałek, and F. M. Peeters, EPL 82, 47002 (2009).

27 B. Szafran, S. Bednarek, M. Dudziak, Phys. Rev. B 75, 235323 (2007).

28 P. F. Bagwell, Phys. Rev. B 41, 10354 (1990). 